\title{
Thermodynamic Principles of Ideal Solid Account for Why Ice Melts at 273.15K
}

\author{
Kentaro Onizuka \\ Thermopyle Co. Ltd., Kyoto, Japan
}

\begin{abstract}
Thermodynamic principles of ideal solid are proposed. Here an ideal solid has perfectly harmonic lattice vibrations. Verifications of the total solution for the Schrödinger equation of a solid revealed a fatal defect in the Debye model. That is, lattice vibrations at higher frequencies than the highest frequency $\omega_{\mathrm{H}}$ allowed in the solid would emerge immediately above $0 \mathrm{~K}$. The principles proposed in this paper fix that fatal defect by introducing thermal expansion to perfectly harmonic vibrations which relaxes those quantum harmonic oscillations excited to a higher eigen energy state than $\hbar \omega_{\mathrm{H}}$, where $\hbar$ is the reduced Planck constant. Thermal expansion, thereby, represses higher frequency vibrations which would inevitably emerge in the Debye model. The pressure for expansion reaches the upper limit at the melting point, and then the solid melts. Hence, the principles account for why ice melts at $273.15(\mathrm{~K}) \simeq \hbar \omega_{\mathrm{H}} /\left(K_{\mathrm{B}} \ln 2\right)=\theta_{\mathrm{D}} / \ln 2$, where $K_{\mathrm{B}}$ is the Boltzmann constant, $\theta_{\mathrm{D}}$ is the Debye temperature by definition, and $\omega_{\mathrm{H}}$ is the highest lattice vibration frequency in ice calculated from the molecular mass, spacing, and the speeds of sound for longitudinal and transverse waves in ice.
\end{abstract}

\section{Introduction}

Modern quantum mechanics began with Schrödinger equation coming out in 1926[1] following de Broglie's hypothesis of matter wave in 1924. The 
models and theories of solid in terms of Planck's classical quantum mechanics had been already known by 1914, a decade before 1926. Those include Lindemann's criterion of melting[2], Einstein solid[3], and Debye model[4].

Surprisingly, however, the Debye model, a representative and long surviving solid model among those known before 1926, had not yet been corroborated by the modern quantum mechanics after 1926. The model obviously has a fatal defect which I found through the verification of the total solution of the Schrödinger equation, the sum of eigen wave functions over all normal modes in a solid. As is detailed in appendices, the sum of two eigen wave functions, $\psi_{A}, \psi_{B}$ in different normal modes would produce a lattice vibration at a frequency higher than the highest frequency $\omega_{\mathrm{H}}$ allowed in the solid if the difference between eigen energy $E_{A}$ of $\psi_{A}$ and that $E_{B}$ of $\psi_{B}$ were larger than $\hbar \omega_{\mathrm{H}}$, since the vibration frequency of the mixed wave function is equal to $\left|\left(E_{\mathrm{A}}-E_{\mathrm{B}}\right) / \hbar\right|$, where $\hbar(=h / 2 \pi)$ is the reduced Planck constant. Consider a case in which eigen function $\psi_{A}$ is in the ground state (quantum number is zero) in the mode of the lowest frequency $\omega_{\mathrm{L}}\left(\ll \omega_{\mathrm{H}}\right)$, and $\psi_{B}$ is in the first excitation state (quantum number is 1) in the mode of the highest frequency $\left(=\omega_{\mathrm{H}}\right)$. The lattice vibration frequency $\simeq 1.5 \times \omega_{\mathrm{H}}$ of the mixed wave function is nearly $50 \%$ higher than $\omega_{\mathrm{H}}$. This means, solid would melt immediately above $0 \mathrm{~K}$ due to emerging vibrations at frequencies higher than the highest frequency $\omega_{\mathrm{H}}$ unless solid has a certain mechanism which relaxes such over-excited oscillations and represses higher frequency vibrations.

Here comes the thermal expansion which had long since been attributed to non-harmonic oscillations. Thermal expansion relaxes those over-excited harmonic oscillations and represses higher frequency lattice vibrations. Lattice vibrating at higher frequencies than $\omega_{\mathrm{H}}$ has the higher frequency components of vibrations which would make particles (atoms or molecules) in solid move more like particles in gas phase confined in a small region, and those particles have strong outward impact when they change directions to move, causing pressure for the solid to expand. Hence, ideal solid without non harmonic oscillation does expand. Then, those oscillations excited to a higher energy state are relaxed and fall to a lower eigen energy state. However, the pressure for expansion has an upper limit as is detailed in appendices. The point of temperature $T_{\mathrm{M}}$ at which the pressure reaches the upper limit is,

$$
T_{\mathrm{M}}=\frac{\hbar \omega_{\mathrm{H}}}{K_{\mathrm{B}} \ln 2}
$$

where $K_{\mathrm{B}}$ is the Boltzmann constant. The Debye temperature $\theta_{\mathrm{D}}$ by defini- 
tion is given as $\theta_{\mathrm{D}}=\hbar \omega_{\mathrm{H}} / K_{\mathrm{B}}$. The melting point is, thereby, $T_{\mathrm{M}}=\theta_{\mathrm{D}} / \ln 2$. Above $T_{\mathrm{M}}$, those excited oscillations to higher energy states are no longer relaxed, and avalanche of excitation to higher energy states absorbing the enthalpy of fusion destroys the lattice structure and then the solid melts, thus the melting point is $T_{\mathrm{M}}$ given in Eq.1.

\section{Results}

Here I show that the melting point of hexagonal ice $\left(\mathrm{I}_{\mathrm{h}}\right)$ calculated by Eq. 1 is almost exactly equal to $273.15(\mathrm{~K})$. Let $C_{\text {Tot }}$ be the total microscopic spring constant and let $m$ be the mass of molecule or atom constituting the solid. The highest frequency $\omega_{\mathrm{H}}$ allowed in the solid is given as,

$$
\omega_{\mathrm{H}}=2 \sqrt{\frac{C_{\mathrm{Tot}}}{m}} .
$$

$C_{\text {Tot }}$ is calculated from the microscopic spring constant $C_{\text {long }}$ for longitudinal wave, and that $C_{\text {trans }}$ for transverse wave (microscopic elastic constant for shear stress) as,

$$
C_{\text {Tot }}=\sqrt{C_{\text {long }}^{2}+2 C_{\text {trans }}^{2}},
$$

where $C_{\text {long }}$ and $C_{\text {trans }}$ are calculated from the speed of sound $c_{\text {long }}$ in solid for longitudinal wave, and that $c_{\text {trans }}$ for transverse wave respectively, and molecular (or atomic) mass $m$, and the average molecular spacing $a$ in solid, For ice (hexagonal solid water $I_{h}$ ), these are given as follows[5],

$$
\begin{aligned}
& m=18.0153(\mathrm{~g} / \mathrm{mol}) \quad=2.99151 \times 10^{-26} \quad(\mathrm{~kg}) \\
& \rho=0.9168 / 0.99997\left(\mathrm{~g} / \mathrm{cm}^{3}\right)=0.91683 \times 10^{3} \quad\left(\mathrm{~kg} / \mathrm{m}^{3}\right) \\
& a=\sqrt[3]{\frac{\rho}{m}} \quad=3.19547 \times 10^{-10} \quad(\mathrm{~m}) \\
& c_{\text {long }}=a \sqrt{\frac{C_{\text {long }}}{m}} \quad=3.230 \quad \times 10^{3} \quad(\mathrm{~m} / \mathrm{s}) \\
& c_{\text {trans }}=a \sqrt{\frac{C_{\text {trans }}}{m}} \quad=1.600 \quad \times 10^{3} \quad(\mathrm{~m} / \mathrm{s})
\end{aligned}
$$

The calculated melting point of ice given by Eq.1 is $270.21 \mathrm{~K}$ which is just 3 degrees lower than the actual melting point of ice $273.15 \mathrm{~K}$. The Debye temperature $\theta_{\mathrm{D}}=\hbar \omega_{\mathrm{H}} / K_{\mathrm{B}}$ of ice is $187.29 \mathrm{~K}$ which is within the range of empirical Debye temperatures which, however, varies significantly from $180 \mathrm{~K}$ up to $320 \mathrm{~K}$ depending on the experimental methods and theories.

The melting point of solids of light atoms in general, such as of beryllium, boron, and even carbon, comes in the range $\left(\theta_{\mathrm{D}}<T_{\mathrm{M}}<2 \theta_{\mathrm{D}}\right)$, suggesting 
$T_{\mathrm{M}}=\theta_{\mathrm{D}} / \ln 2$, though for heavy atoms, $T_{\mathrm{M}}$ is sometimes much higher than $2 \theta_{\mathrm{D}}$ presumably because the actual Debye temperature is much higher than that estimated from the heat capacity at low temperature.

\section{Discussion}

I propose thermodynamic principles of ideal solid based on those abovementioned findings.. Here, ideal solid is such that lattice vibrations are harmonic. And I will show that the heat capacity of solid is not drastically different from that in Debye model.

The principle of lattice vibration. Lattice vibrations in solid are different from the quantum harmonic oscillations each in a normal mode, but are derived from the sum of eigen wave functions each in the different normal mode. Here the mixture of two wave functions has the vibrating expectation value for the deviation from the equilibrium position of the lattice as is given in Eq. 16 in appendices.

The principle of thermal expansion. Those oscillations excited to a state of higher eigen energy than $\hbar \omega_{\mathrm{H}}$ are relaxed by the thermal expansion as the relaxation transfers the energy for excitation to the energy for thermal expansion, the elastic energy stored in the stretched molecular (or atomic) spacing.

The principle of melting. In each normal mode the energy transferred to the thermal expansion has an upper limit $\simeq \hbar \omega_{\mathrm{H}}$ which is derived from the partition functions for the repressed harmonic oscillator in which excitation to higher energy states than $\hbar \omega_{\mathrm{H}}$ is repressed (see Eq. 22,23, and 24 in appendices). In other words, the pressure for the solid to thermally expand has an upper limit. When the rising temperature exceeds $T_{\mathrm{M}}=\hbar \omega_{\mathrm{H}} /\left(K_{\mathrm{B}} \ln 2\right)$ at which the pressure for thermal expansion reaches the limit, the excitation to higher energy states is no longer repressed because the solid cannot further expand, and avalanche of excitation released above $T_{\mathrm{M}}$ absorbs enthalpy of fusion and then the solid melts.

Heat capacity of ideal solid. These three principles give the isobaric heat capacity of ideal solid which is nothing but the isochoric heat capacity derived in the Debye model with just a slight modification due to the different dispersion relations (Debye approximation). See appendices for detail. 


\section{Conclusion}

I have three points to indicate as concluding remarks.

The principles of ideal solid in this paper give a clear image of what liquid in general could be like in terms of quantum mechanics. Excitation to higher energy states than $\hbar \omega_{\mathrm{H}}$ is no longer repressed in liquid, but excitation to higher energy states than the depth of the force field potentials among particles in liquid should be relaxed, because those particles whose energy is higher than the potential depth produce vapor pressure. When vapor pressure exceeds ambient pressure, the liquid boils.

Since the excitation to higher energy states than $\hbar \omega_{\mathrm{H}}$ is repressed for all quantum harmonic oscillators in solid, excitation would never occur in those modes whose frequency $\omega$ is higher than $2 \omega_{\mathrm{H}} / 3$ below the melting point. Considering the significantly large share of those high frequency modes in solid, the population of phonons must be much smaller than that has ever been expected. Some theories relevant to phonons in solid might need drastic revisions.

The principles of melting in this paper must work in other phenomena of melting in broader sense, such as thermal denaturation and dissociation of biomolecules (e.g. proteins, DNA, RNA, and sugar). And also, chemical reactions including dissolution in condensed state would be ruled by those principles, as there must be low frequency vibrations in background, which produce higher frequency vibrations than that allowed in chemical bonds.

\section{Appendices}

\section{The principle of lattice vibration}

Consider an ideal solid consisting of $N$ atoms. Each atom has three degrees of freedom each for the motion in the direction of mutually orthogonal $X, Y$ or $Z$ axis. Hence the solid has in total $3 N$ degrees of freedom $(N$ degrees in each direction), and has $3 N$ normal modes each of which is a quantum harmonic oscillator in the three-dimensional ideal solid. The Schrödinger equation for a mode of wave number vector $\boldsymbol{k}$ of motions in the direction of $X$ axis (deviation $x$ in the direction of $X$ axis) is given as,

$$
i \hbar \frac{\partial}{\partial t}|\psi\rangle=\hat{H}_{\boldsymbol{k}}^{X}|\psi\rangle
$$


where $\hat{H}_{k}^{X}$ is the Hamiltonian for the mode, and $\psi$ is the wave function. The eigen function of this equation for the harmonic oscillator is,

$$
\psi_{\boldsymbol{k}}^{X}\left(n_{\boldsymbol{k}}^{X}\right)=\psi_{\boldsymbol{k}}^{X}\left(n_{\boldsymbol{k}}^{X}, x\right) e^{-i \omega_{\boldsymbol{k}}^{X}\left(n_{\boldsymbol{k}}^{X}\right) t}
$$

where $x$ denotes the deviation from the equilibrium of lattice in direction $X$, and the frequency, $\omega_{\boldsymbol{k}}^{X}\left(n_{X}^{\boldsymbol{k}}\right)$, is given from the normal mode frequency $\omega_{\boldsymbol{k}}^{X}$ of the oscillator and quantum number $n_{\boldsymbol{k}}^{X}=0,1,2, \ldots$,

$$
\omega_{\boldsymbol{k}}^{X}\left(n_{\boldsymbol{k}}^{X}\right)=\left(\frac{1}{2}+n_{k}^{X}\right) \omega_{\boldsymbol{k}}^{X}
$$

The eigen energy $E_{\boldsymbol{k}}^{X}\left(n_{\boldsymbol{k}}^{X}\right)$ is, then, given as,

$$
E_{\boldsymbol{k}}^{X}\left(n_{\boldsymbol{k}}^{X}\right)=\hbar \omega_{\boldsymbol{k}}^{X}\left(n_{\boldsymbol{k}}^{X}\right)=\hbar\left(\frac{1}{2}+n_{\boldsymbol{k}}^{X}\right) \omega_{\boldsymbol{k}}^{X}
$$

The total Hamiltonian $\hat{H}_{\text {Tot }}$ for the ideal solid is the sum of Hamiltonians over all modes and directions, therefore,

$$
\hat{H}_{\mathrm{Tot}}=\sum_{\boldsymbol{k}}\left[\hat{H}_{\boldsymbol{k}}^{X}+\hat{H}_{\boldsymbol{k}}^{Y}+\hat{H}_{\boldsymbol{k}}^{Z}\right] .
$$

Here, Hamiltonian in a direction operated to a wave function in another direction is always zero, and also Hamiltonian in a mode $\boldsymbol{k}$ operated to a wave function in another mode $\boldsymbol{l}(\neq \boldsymbol{k})$ is always zero,

$$
\begin{aligned}
& \hat{H}^{X} \psi^{Y} \equiv 0, \quad \hat{H}^{X} \psi^{Z} \equiv 0, \quad \hat{H}^{Y} \psi^{Z} \equiv 0, \\
& \hat{H}^{Y} \psi^{X} \equiv 0, \quad \hat{H}^{Z} \psi^{X} \equiv 0, \quad \hat{H}^{Z} \psi^{Y} \equiv 0, \\
& \hat{H}_{\boldsymbol{k}}^{X} \psi_{\boldsymbol{l}}^{X} \equiv 0, \quad \hat{H}_{\boldsymbol{k}}^{Y} \psi_{\boldsymbol{l}}^{Y} \equiv 0, \quad \hat{H}_{\boldsymbol{k}}^{Z} \psi_{\boldsymbol{l}}^{Z} \equiv 0 .
\end{aligned}
$$

Hence, the total wave function is simply the sum of eigen functions over all modes and directions, and also the total energy is simply the sum of eigen energies,

$$
\begin{aligned}
\psi_{\mathrm{Tot}} & =\sum_{\boldsymbol{k}}\left[\psi_{\boldsymbol{k}}^{X}\left(n_{\boldsymbol{k}}^{X}\right)+\psi_{\boldsymbol{k}}^{Y}\left(n_{\boldsymbol{k}}^{Y}\right)+\psi_{\boldsymbol{k}}^{Z}\left(n_{\boldsymbol{k}}^{Z}\right)\right] \\
E_{\mathrm{Tot}} & =\sum_{\boldsymbol{k}}\left[E_{\boldsymbol{k}}^{X}\left(n_{\boldsymbol{k}}^{X}\right)+E_{\boldsymbol{k}}^{Y}\left(n_{\boldsymbol{k}}^{Y}\right)+E_{\boldsymbol{k}}^{Z}\left(n_{\boldsymbol{k}}^{Z}\right)\right] .
\end{aligned}
$$

The total Schrödinger equation for ideal solid is, thereby,

$$
i \hbar \frac{\partial}{\partial t}\left|\psi_{\text {Tot }}\right\rangle=E_{\text {Tot }}\left|\psi_{\text {Tot }}\right\rangle=\hat{H}_{\text {Tot }}\left|\psi_{\text {Tot }}\right\rangle
$$


Hence, the total solution for ideal solid must include sum of eigen functions each in different mode.

Now we consider why the lattice in solid vibrates in real space. In quantum mechanics, any observable value $\xi$ is calculated as the expectation value $\langle\psi|\xi| \psi\rangle$ from the wave function $\psi$. So is the deviation from the equilibrium of lattice, $x, y$, or $z$, as,

$$
\langle x\rangle=\left\langle\psi^{X}|x| \psi^{X}\right\rangle,\langle y\rangle=\left\langle\psi^{Y}|y| \psi^{Y}\right\rangle,\langle z\rangle=\left\langle\psi^{Z}|z| \psi^{Z}\right\rangle,
$$

where,

$$
\psi^{X}=\sum_{\boldsymbol{k}} \psi_{\boldsymbol{k}}^{X}\left(n_{\boldsymbol{k}}^{X}\right), \psi^{Y}=\sum_{\boldsymbol{k}} \psi_{\boldsymbol{k}}^{Y}\left(n_{\boldsymbol{k}}^{Y}\right), \psi^{Z}=\sum_{\boldsymbol{k}} \psi_{\boldsymbol{k}}^{Z}\left(n_{\boldsymbol{k}}^{Z}\right) .
$$

Here we calculate the expectation value $\langle x\rangle$ of the sum of just two wave functions, $\psi_{\boldsymbol{k}}^{X}\left(n_{\boldsymbol{K}}^{X}\right)$ and $\psi_{\boldsymbol{l}}^{X}\left(n_{\boldsymbol{l}}^{X}\right)$ of different modes, $\boldsymbol{k} \neq \boldsymbol{l}$ in the same direction $X$. The expectation value is given as follows,

$$
\begin{aligned}
\langle x\rangle & =\left\langle\psi_{\boldsymbol{k}}^{X}\left(n_{\boldsymbol{k}}^{X}\right)+\psi_{\boldsymbol{l}}^{X}\left(n_{\boldsymbol{l}}^{X}\right)|x| \psi_{\boldsymbol{k}}^{X}\left(n_{\boldsymbol{k}}^{X}\right)+\psi_{\boldsymbol{l}}^{X}\left(n_{\boldsymbol{l}}^{X}\right)\right\rangle \\
& =\left\langle\psi_{\boldsymbol{k}}^{X}\left(n_{\boldsymbol{k}}^{X}\right)|x| \psi_{\boldsymbol{l}}^{X}\left(n_{\boldsymbol{l}}^{X}\right)\right\rangle+\left\langle\psi_{\boldsymbol{l}}^{X}\left(n_{\boldsymbol{l}}^{X}\right)|x| \psi_{\boldsymbol{k}}^{X}\left(n_{\boldsymbol{k}}^{X}\right)\right\rangle \\
& =A\left(\boldsymbol{k}, \boldsymbol{l}, n_{\boldsymbol{k}}^{X}, n_{\boldsymbol{l}}^{X}\right) \cos \left(\omega_{\mathrm{Mix}}^{X} t\right),
\end{aligned}
$$

where $A\left(\boldsymbol{k}, \boldsymbol{l}, n_{\boldsymbol{k}}^{X}, n_{\boldsymbol{l}}^{X}\right)$ is the amplitude, and $\omega_{\text {Mix }}^{X}$ is the frequency of the vibration of the expectation value for deviation $x$ in direction $X$. Amplitude $A\left(\boldsymbol{k}, \boldsymbol{l}, n_{X}^{\boldsymbol{k}}, n_{X}^{\boldsymbol{l}}\right)$ is zero if the sum of two quantum numbers, $n_{\boldsymbol{k}}^{X}+n_{\boldsymbol{l}}^{X}$, is an even number $(0,2,4, \ldots)$, but is not zero if an odd number $(1,3,5,7, \ldots)$. Here $\omega_{\text {Mix }}^{X}$ is given as,

$$
\omega_{\text {Mix }}^{X}=\left|\omega_{\boldsymbol{k}}^{X}\left(n_{\boldsymbol{k}}^{X}\right)-\omega_{\boldsymbol{l}}^{X}\left(n_{\boldsymbol{l}}^{X}\right)\right|
$$

The expectation value for the deviation from the equilibrium position in the lattice actually vibrate at the frequency $\omega_{\text {Mix }}^{X}$.

\section{The principle of thermal expansion}

The vibration frequency $\omega_{\text {Mix }}^{X}$ in Eq. 17 could obviously exceed $\omega_{\mathrm{H}}$ if $\mid \omega_{\boldsymbol{k}}^{X}\left(n_{\boldsymbol{k}}^{X}\right)-$ $\omega_{l}^{X}\left(n_{l}^{X}\right) \mid$ were greater than $\omega_{\mathrm{H}}$. The excitation to such a state whose eigen energy is higher than $\hbar \omega_{\mathrm{H}}$ must be relaxed in solid by the thermal expansion. Here, we calculate the partition function $Z_{\mathrm{RHO}}(\omega)$ of a repressed harmonic oscillator (RHO) whose frequency is $\omega\left(\leq \omega_{\mathrm{H}}\right)$, and whose highest eigen energy is set to no higher than $\hbar \omega_{\mathrm{H}}$. The largest quantum number $n_{\text {Max }}$ allowed 
in this repressed harmonic oscillator is determined so that,

$$
\omega\left(\frac{1}{2}+n_{\mathrm{Max}}\right)<\omega_{\mathrm{H}}<\omega\left(\frac{1}{2}+n_{\mathrm{Max}}+1\right) .
$$

Thereby,

$$
Z_{\mathrm{RHO}}(\omega)=\sum_{n=0}^{n_{\mathrm{Max}}} e^{-\hbar \omega(1 / 2+n) / K_{\mathrm{B}} T}=Z_{\mathrm{SHO}}(\omega)-Z_{\mathrm{R}}(\omega)
$$

where $Z_{\mathrm{SHO}}(\omega)$ is the partition function for a simple harmonic oscillator, and $Z_{\mathrm{R}}(\omega)$ is that for repression, given as,

$$
\begin{aligned}
Z_{\mathrm{SHO}}(\omega) & =\frac{1}{2 \sinh \left(\hbar \omega / 2 K_{\mathrm{B}} T\right)}, \\
Z_{\mathrm{R}}(\omega) & =\frac{e^{-\hbar \omega\left(n_{\mathrm{Max}}+1\right) / K_{\mathrm{B}} T}}{2 \sinh \left(\hbar \omega / 2 K_{\mathrm{B}} T\right)} .
\end{aligned}
$$

Here, $Z_{\mathrm{R}}$ is the very partition function for thermal expansion.

\section{The principle of melting}

The partition functions $Z_{\mathrm{RHO}}(\omega)$ and $Z_{\mathrm{R}}(\omega)$ determines the melting point as follows. Here, note that $Z_{\mathrm{RHO}}(\omega)$ must be greater than $Z_{\mathrm{R}}(\omega)$ for repressed harmonic oscillator, $Z_{\mathrm{RHO}}(\omega)>Z_{\mathrm{R}}(\omega)$, because the probability, $P_{\mathrm{Ex}}=Z_{\mathrm{R}}(\omega) / Z_{\mathrm{SHO}}(\omega)$, of the oscillation being excited from a state whose eigen energy is lower than $\hbar \omega_{\mathrm{H}}$ to a higher energy state is always smaller than the probability, $P_{\mathrm{RHO}}=Z_{\mathrm{RHO}}(\omega) / Z_{\mathrm{SHO}}(\omega)$, of the oscillation being in a lower energy state than $\hbar \omega_{\mathrm{H}}$ for a repressed harmonic oscillator. Then,

$$
Z_{\mathrm{RHO}}(\omega)-Z_{\mathrm{R}}(\omega)=\frac{1-2 e^{-\hbar \omega\left(n_{\mathrm{Max}}+1\right) / K_{\mathrm{B}} T}}{2 \sinh \left(\hbar \omega / 2 K_{\mathrm{B}} T\right)}>0 .
$$

The critical temperature $T_{\mathrm{C}}$ at which $Z_{\mathrm{RHO}}(\omega)-Z_{\mathrm{R}}(\omega)=0$ is given as,

$$
T_{\mathrm{C}}=\left(\frac{1}{\ln 2}\right) \frac{\hbar \omega\left(n_{\mathrm{Max}}+1\right)}{K_{\mathrm{B}}} .
$$

For most modes, $\omega\left(n_{\mathrm{Max}}+1\right) \simeq \omega_{\mathrm{H}}$, thus, the critical temperature $T_{\mathrm{C}}$ is considered the melting point $T_{\mathrm{M}}$,

$$
T_{\mathrm{M}}=\left(\frac{1}{\ln 2}\right) \frac{\hbar \omega_{\mathrm{H}}}{K_{\mathrm{B}}} .
$$


This is exactly identical to Eq. 1. Above $T_{\mathrm{M}}$, excitation to higher energy states than $\hbar \omega_{\mathrm{H}}$ is no longer repressed because outward pressure for expansion reaches the upper limit due to $Z_{\mathrm{R}}(\omega)>Z_{\mathrm{RMO}}(\omega)$ in most modes.

Here we calculate the mean energy $\left\langle E_{\mathrm{RHO}}(\omega)\right\rangle$ of repressed harmonic oscillator. Let $\beta$ be $1 / K_{\mathrm{B}} T$. The mean energy, $\left\langle E_{\mathrm{RHO}}(\omega)\right\rangle$, is calculated from the partition function, $Z_{\mathrm{RHO}}(\omega)$, as follows,

$$
\left\langle E_{\mathrm{RHO}}(\omega)\right\rangle=-\frac{1}{Z_{\mathrm{RHO}}(\omega)} \frac{\partial}{\partial \beta} Z_{\mathrm{RHO}}(\omega)=\left\langle E_{\mathrm{SHO}}(\omega)\right\rangle-\left\langle E_{\mathrm{R}}(\omega)\right\rangle
$$

where,

$$
\begin{aligned}
\left\langle E_{\mathrm{SHO}}(\omega)\right\rangle & =\left(\frac{\hbar \omega}{2}\right) \operatorname{coth}\left(\frac{\hbar \omega}{2 K_{\mathrm{B}} T}\right) \\
\left\langle E_{\mathrm{R}}(\omega)\right\rangle & =\left(\frac{\hbar \omega\left(n_{\mathrm{Max}}+1\right)}{2}\right) \frac{e^{-\hbar \omega\left(n_{\mathrm{Max}}+1\right) / 2 K_{\mathrm{B}} T}}{\sinh \left(\frac{\hbar \omega\left(n_{\mathrm{Max}}+1\right)}{2 K_{\mathrm{B}} T}\right)} .
\end{aligned}
$$

Here, $\left\langle E_{\mathrm{SHO}}(\omega)\right\rangle$ is the mean energy of an ordinary simple harmonic oscillator in which no excitation is repressed, and $\left\langle E_{\mathrm{R}}(\omega)\right\rangle$ is the energy transferred to the elastic energy in repressing excitation to higher energy states than $\hbar \omega_{\mathrm{H}}$. Since $Z_{\mathrm{RHO}}(\omega)>Z_{\mathrm{R}}(\omega)$ as mentioned above, the upper limit of $\left\langle E_{\mathrm{R}}(\omega)\right\rangle$ is $\hbar \omega\left(n_{\mathrm{Max}}+1\right)$ around the melting point.

\section{Isobaric heat capacity of solid}

The isobaric heat capacity $C_{\mathrm{P}}$ of solid is similar to isochoric heat capacity $C_{\mathrm{V}}^{\mathrm{D}}$ in Debye model, as for each mode whose frequency is $\omega$,

$$
\begin{aligned}
C_{\mathrm{P}}(\omega) & =\frac{\partial}{\partial T}\left[\left\langle E_{\mathrm{RHO}}(\omega)\right\rangle+\left\langle E_{\mathrm{R}}(\omega)\right\rangle\right]=\frac{\partial}{\partial T}\left\langle E_{\mathrm{SHO}}(\omega)\right\rangle \\
& =C_{\mathrm{V}}^{\mathrm{D}}(\omega)=K_{\mathrm{B}}\left(\frac{\hbar \omega}{2 K_{\mathrm{B}} T}\right)^{2}\left(\frac{1}{\sinh \left(\frac{\hbar \omega}{2 K_{\mathrm{B}} T}\right)}\right)^{2} .
\end{aligned}
$$

Hence, the isobaric heat capacity $C_{\mathrm{P}}$ of solid derived from the principles of ideal solid agrees with isochoric heat capacity $C_{\mathrm{V}}^{\mathrm{D}}$ in Debye model with a slight modification due to the different dispersion relations (Debye approximation) employed in the model.

$$
\omega=\sin \left(\frac{k a}{2}\right) \sqrt{\frac{C_{\mathrm{Tot}}}{m}} \neq k a \sqrt{\frac{C_{\mathrm{Tot}}}{m}} .
$$


where $k$ is the wave number. Debye model is accurate enough, but essentially wrong because it ignores thermal expansion and melting of solid.

\section{Acknowledgments}

I thank Shinichi Yamabe, professor emeritus at Nara University of Education, who carefully reviewed the draft of this paper. I thank Shigeo Yoshii at Panasonic for discussions in the early stage of my study at ATRL(Panasonic) until Sep. 2014, and AIS(Panasonic) until Feb. 2016.

\section{References}

[1] Schrödinger E, An Undulatory Theory of the Mechanics of Atoms and Molecules, Physical Review 1926, 28(6):1049-1070.

[2] Lindemann F-A, Phys. Z 1910, 11:609-614.

[3] Einstein A, Theorie der Strahlung und die Theorie der Spezifischen Wärme, Analen der Physik 1907, 4,22:180-190,800.

[4] Debye P, Zur Theorie der spezifischen Wärme, Annalen der Physik 1912, 39(4):789-839.

[5] Chronological Scientific Tables, 2017, National Astronomical Observatory of Japan. 